\title{
2-S20-1 Symposium
}

\section{Possible contribution of PACAP-evoked spinal astrocyte-neuron lactate shuttle to the chronic pain development}

\author{
Takashi Kurihara $^{1}$, Yuki Kambe ${ }^{1}$, Masafumi Yokai ${ }^{1}$, Ayaka Shimodaira ${ }^{2}$, Ichiro Takasaki ${ }^{2}$, \\ Atsuro Miyata ${ }^{1}$
}

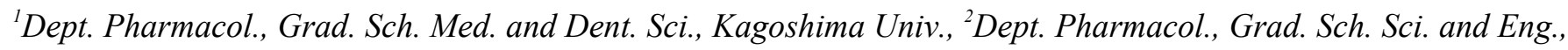
Univ. of Toyama

Previously, we showed that spinal pituitary adenylate cyclase-activating polypeptide (PACAP)/PAC1 receptor signaling triggers long-lasting pain-like behaviors through astroglial activation. Since astrocyte-neuron lactate shuttle (ANLS) could be essential for long-term synaptic plasticity, we aimed to elucidate a possible involvement of spinal ANLS in the development of the PACAP-evoked pain-like behaviors. A single intrathecal administration of PACAP induced short-term spontaneous aversive behaviors, followed by long-lasting mechanical allodynia in mice. These behaviors were inhibited by $\mathrm{DAB}$, an inhibitor of glycogenolysis, and this inhibition was reversed by simultaneous Llactate application. In the cultured spinal astrocytes, the PACAP-evoked glycogenolysis and lactate secretion were inhibited by a protein kinase C (PKC) inhibitor, and the PKC inhibitor attenuated the PACAP-induced pain-like behaviors. Moreover, an inhibitor for the monocarboxylate transporters blocked the lactate secretion from the spinal astrocytes and inhibited the PACAP-evoked pain-like behaviors. In this symposium, we will further discuss possible involvement of the spinal PACAP-ANLS signaling in an experimental model of neuropathic pain. 